\title{
Shot Noise by Quantum Scattering in Chaotic Cavities
}

\author{
S. Oberholzer, E. V. Sukhorukov, C. Strunk,* and C. Schönenberger \\ Institut für Physik, Universität Basel, Klingelbergstrasse 82, CH-4056 Basel, Switzerland \\ T. Heinzel \\ Solid State Physics Laboratory, ETH-Zürich, CH-8093 Zürich, Switzerland \\ M. Holland \\ Department of Electronics, University of Glasgow, Glasgow G12 8QQ, United Kingdom
}

(Received 6 September 2000)

\begin{abstract}
We have experimentally studied shot noise of chaotic cavities defined by two quantum point contacts in series. The cavity noise is determined as $(1 / 4) 2 e|I|$ in agreement with theory and can be well distinguished from other contributions to noise generated at the contacts. Subsequently, we have found that cavity noise decreases if one of the contacts is further opened and reaches nearly zero for a highly asymmetric cavity. Heating inside the cavity due to electron-electron interaction can slightly enhance the noise of large cavities and is also discussed quantitatively.
\end{abstract}

The nonequilibrium time dependent fluctuations of the electrical current, known as shot noise, are caused by the randomness of charge transfer in units of $e$ [1]. If the electron transfer can be described by a Poissonian process, the spectral density $S$ of the current fluctuations is $S_{\text {Poisson }}=2 e|I|$. Correlations imposed by fermionic statistics as well as Coulomb interaction may change shot noise from $S_{\text {Poisson. This is expressed by the Fano factor }}$ $F$ defined as $F \equiv S / S_{\text {Poisson. A quantum wire with an }}$ intermediate barrier with energy-independent transmission probability $\Gamma, \Gamma=1 / 2$, for example, has a Fano factor of $1-\Gamma=1 / 2[2,3]$. This suppression is due to binominal instead of Poissonian statistics. Here, we explore what happens if the barrier is replaced by a chaotic cavity [Fig. 1, inset (a)]. For a symmetric and open cavity, which is a cavity connected to ideal reservoirs via two identical noiseless (barrier-free) quantum wires, the mean transmission probability is $1 / 2$, too. But surprisingly, the Fano factor is predicted to be only $1 / 4$ [4,5]. The 1/4 Fano factor, valid for open and symmetric chaotic cavities, was first derived by random matrix theory, which is based on phase-coherent quantum mechanical transport [4]. Recently, a semiclassical analysis using the "principle of minimal correlations" arrived at the same result [5].

Similar to metallic diffusive wires, where $F=1 / 3$ [6-8], the Fano factor $1 / 4$ for chaotic cavity is universal in the sense that it is insensitive to microscopic properties $[4,5,9,10]$. Nevertheless, there is an important difference between these two systems concerning the origin of resistance and noise. In a diffusive conductor resistance and shot noise are both generated locally at scattering centers, which are homogeneously distributed along the wire. In an open chaotic cavity resistance and shot noise are generated differently. The resistance is due to the fundamental quantum resistance of the contacts. Although the source of resistance, the open contacts do not con- tribute to noise because electrons are transmitted with unit probability. Shot noise arises inside the cavity due to quantum mechanical diffraction which splits the electron wave packet into two partial waves leaving the two exits. In the semiclassical approach cavity noise is determined by the average fluctuations of the state occupancy inside the cavity given, at $T=0$, by [5]

$$
S=2 G \int d E f_{C}\left(1-f_{C}\right) .
$$

Here $f_{C}(E)$ denotes the distribution function inside the cavity, which is homogeneous and isotropic. The total conductance $G=G_{0}\left(N_{L} N_{R}\right) /\left(N_{L}+N_{R}\right)$ with $G_{0}=2 e^{2} / h$ is equal to the series conductance of the left and right

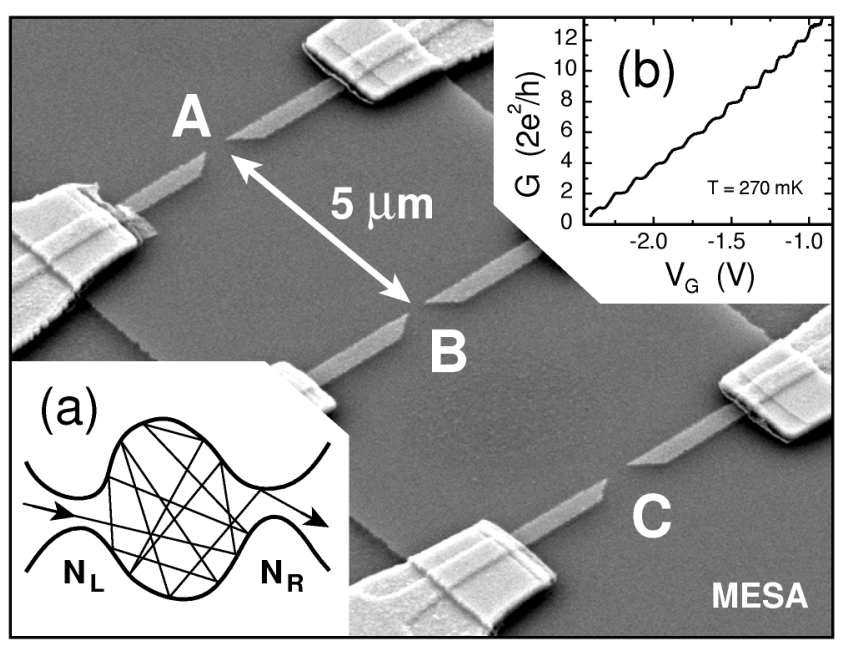

FIG. 1. Scanning electron microscope picture of a Hall bar with three QPCs in series used to define chaotic cavities of different size. (a) The ratio of the number of modes $\eta \equiv N_{L} / N_{R}=$ $G_{L} / G_{R}$ can be adjusted by varying the openings of the left and right contacts, independently. (b) QPC conductance vs gate voltage of one of the contacts. 
contacts with $N_{L}\left(N_{R}\right)$ open channels (i.e., $\Gamma_{1, \ldots, N_{L, R}}=1$, $\Gamma_{>N_{L, R}}=0$ ). For noninteracting electrons the distribution function in the cavity $f_{C}$ just equals the weighted average of the distribution functions $f_{L}$ and $f_{R}$ in the left and right reservoirs. In the symmetric case $N_{L}=N_{R}$, i.e., $f_{C}=\frac{1}{2}\left(f_{L}+f_{R}\right)$, Eq. (1) yields a Fano factor of $1 / 4$. For very asymmetric contacts $\left(N_{L} \gg N_{R}\right)$ shot noise approaches zero, since the system can then be regarded as a single contact with $N_{R}$ open and therefore noiseless channels. The general Fano factor $F \equiv S / 2 e|I|$ for cavity noise is

$$
F(\eta)=\frac{N_{L} N_{R}}{\left(N_{L}+N_{R}\right)^{2}}=\frac{\eta}{(1+\eta)^{2}},
$$

where we introduce the parameter $\eta \equiv N_{L} / N_{R}$ measuring the symmetry of the cavity.

Experimentally, we have realized chaotic cavities by two quantum point contacts (QPC) in series. These are electrostatically defined in a two dimensional electron gas (2DEG) by metallic split gates on top (see Fig. 1) [11]. The opening of the contacts can be individually tuned by varying the applied gate voltages independently. The 2DEG forms $80 \mathrm{~nm}$ below the surface at the interface of a standard $\mathrm{GaAs} / \mathrm{Al}_{0.3} \mathrm{Ga}_{0.7}$ As heterojunction. Magnetoresistance measurements yield a carrier density of $2.7 \times$ $10^{15} \mathrm{~m}^{-2}$, corresponding to a Fermi energy of $\simeq 106 \mathrm{~K}$ and a mobility of $83 \mathrm{~V} \mathrm{~s} / \mathrm{m}^{-2}$ resulting in a mean free path of $\simeq 7 \mu \mathrm{m}$ comparable to the size of the cavity. Three QPCs in series as shown in Fig. 1 enable one to define two cavities of different size: either the outer gates $\mathrm{A}$ and $\mathrm{C}$ with the middle gate $\mathrm{B}$ kept completely open can be used to define a relatively large cavity of $\simeq 11 \times 8 \mu \mathrm{m}$ or two of the inner gates $(\mathrm{A}, \mathrm{B}$ or $\mathrm{B}, \mathrm{C})$ create a smaller cavity of $\simeq 5 \times 8 \mu \mathrm{m}$. The conductance of the QPCs is quantized according to the Landauer formula $G=G_{0} \sum_{n} \Gamma_{n}$ [12] [inset (b) of Fig. 1]. An open cavity is defined when both QPCs are adjusted to a conductance plateau, where $N$ modes are fully transmitted $(\Gamma=1)$ and the others are totally reflected $(\Gamma=0)$. Since the gates do not influence each other, the transmission of both contacts can be determined independently by completely opening one of them while the conductance of the other is measured. The two-terminal conductance $G$ is experimentally found to correspond to the series conductance of the two contacts $G_{L} G_{R} /\left(G_{L}+G_{R}\right)$ with an accuracy of less than $1 \%$ [4,5]. Therefore, direct transmission of electrons from the left to the right contact can be excluded, as well as quantum corrections [13,14].

Two independent low-noise amplifiers (EG\&G 5184) operating at room temperature are used to detect the voltage fluctuations across the cavity. A spectrum analyzer (HP 89410A) calculates the cross-correlation spectrum of the two amplified signals. This technique allows one to reduce uncorrelated noise contributions which do not originate from the sample itself. Experimental details can be found in $[8,15]$. Furthermore, the whole setup is filtered against RF interference at low temperatures by a shielded sample box and lossy microcoaxes to minimize heating by radiation. Voltage noise is typically measured at frequencies around $6 \mathrm{kHz}$ where the noise is frequency independent (white) up to the maximum bias current $\leq 50 \mathrm{nA}$ used in the experiment. The sensitivity for voltage noise measurements is of the order $5 \times 10^{-21} \mathrm{~V}^{2} \mathrm{~s}$. The measured noise is calibrated against equilibrium Nyquist noise at different bath temperatures. From the Nyquist relation $S_{V}=4 k_{B} R T$ the voltage gain as well as the offset in the voltage noise $S_{I}^{\text {off }} R^{2}$ caused by the finite current noise $S_{I}^{\text {off }}$ of the amplifiers can be determined with high accuracy. Although shot noise is a nonequilibrium phenomenon observed in its purest form in the limit $e V \gg k_{B} T$, in this experiment bias voltages are limited to $\simeq 8 k_{B} T / e$, only. This is to avoid nonlinearities of the current-voltage characteristics of the QPCs [16] and 1/f-noise contributions occurring at larger currents [15]. Within this limit, the differential resistance, recorded for all noise measurements, changes by $\lesssim 2.5 \%$. The current noise is finally obtained from the measured voltage fluctuations by $S_{I}=$ $S_{V} /(d V / d I)^{2}-S_{I}^{\text {off }}$.

Figure 2 shows shot noise measurements of a cavity defined by gates $\mathrm{A}$ and $\mathrm{B}$ with a size of $\simeq 5 \times 8 \mu \mathrm{m}$ for different symmetry parameters $\eta=G_{L} / G_{R}$. The solid curves describe the crossover from thermal to shot noise for the measured value of $\eta$ given by [5]

$$
S=S_{\mathrm{eq}}\left\{1+F(\eta)\left[\frac{e V}{2 k_{B} T} \operatorname{coth}\left(\frac{e V}{2 k_{B} T}\right)-1\right]\right\} .
$$

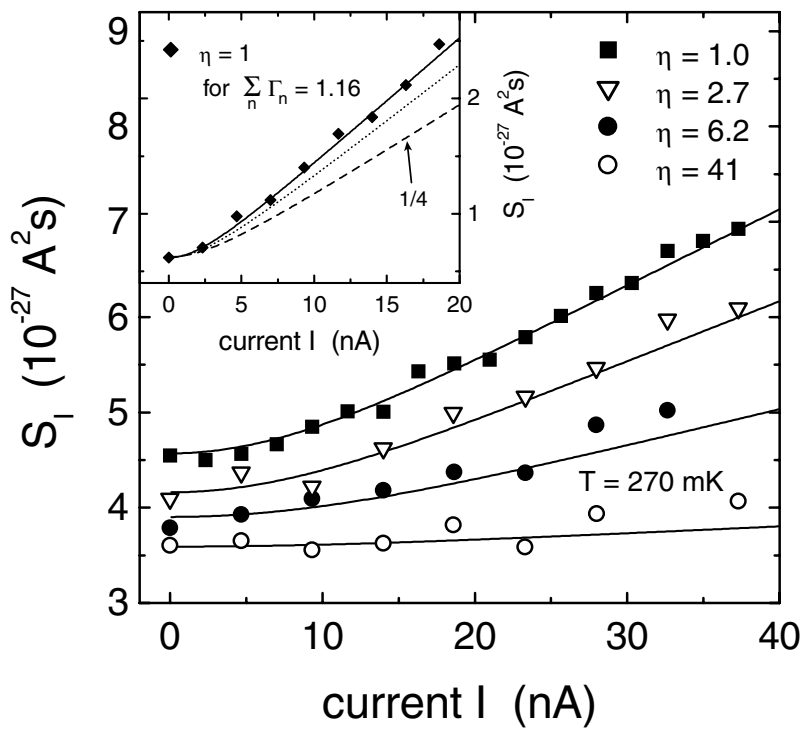

FIG. 2. Shot noise of a chaotic cavity with ideal contacts $\left(G_{L, R} / G_{0}=\right.$ integer $)$ for different conductance ratios $\eta=G_{L} / G_{R}$. The data for $\eta=1.0,2.7$, and 6.2 are offset for clarity by 20,15 , and $5 \times 10^{-28} \mathrm{~A}^{2}$ s, respectively. Inset: Shot noise is larger than $(1 / 4) 2 e|I|$ if there is additional partitioning due to nonideal contacts $\left(G_{L, R} / G_{0} \neq\right.$ integer). The curves are numerical calculations assuming no mode mixing (dotted line) and for slight mode mixing of $10 \%$ (solid line). 
$S_{\text {eq }}=4 k_{B} T G$ denotes the equilibrium noise and $F(\eta)$ the Fano factor [Eq. (2)]. In the symmetric case $(\eta=1)$ with $N_{L}=N_{R}=5$ we obtain a very good agreement between the experimental data and the theoretical prediction of $(1 / 4) 2 e|I|$. When the right contact is further opened $\left(G_{R}>G_{L}\right), \eta$ increases from 1 (symmetric) to $\simeq 41$ (asymmetric). Thereby, shot noise gradually disappears for larger values of $\eta$ as expected from Eq. (2). For partial transmission in the contacts shot noise is larger than $(1 / 4) 2 e|I|$ because additional noise is generated at the contacts. This is shown in the inset of Fig. 2 where the conductance of each point contact equals $1.16 G_{0}$. The dotted curve is a numerical calculation for no mode mixing. The data are approximated the best assuming slight mode mixing of $\simeq 10 \%$ (solid line) with $\Gamma_{1}=0.90$ and $\Gamma_{2}=0.26$.

Up to now we have assumed that inelastic electron scattering inside the cavity can be neglected. In general, heating caused by electron-electron interaction enhances shot noise [1]. The Fano factor of a diffusive wire, for example, changes from $1 / 3$ for noninteracting (cold) electrons to $\sqrt{3} / 4$ for interacting (hot) electrons [17]. Heating also affects the shot noise of a chaotic cavity. The Fano factor is modified to [18]

$$
F(\eta)=\frac{\sqrt{3 N_{L} N_{R}}}{\pi\left(N_{L}+N_{R}\right)}=\frac{\sqrt{3 \eta}}{\pi(1+\eta)},
$$

and the crossover from thermal to shot noise is described by

$$
S=\frac{S_{\mathrm{eq}}}{2}\left\{1+\sqrt{1+F(\eta)^{2}\left(\frac{e V}{k_{B} T}\right)^{2}}\right\} .
$$

For a symmetric cavity $F(\eta=1) \simeq 0.276$ for hot electrons, which is only slightly larger than $F(\eta=1)=0.25$ for cold electrons. The inset of Fig. 3 compares $S\left(e V / k_{B} T\right)$ in the hot and cold electron regime for a diffusive wire and a cavity. As is evident, the differences are very small, in particular, in case of a cavity where even a crossing at $e V / k_{B} T \simeq 15$ occurs. In Fig. 3 the measured noise for $\eta=1$ of Fig. 2 is replotted and compared to the prediction for cold (solid line) and for hot electrons (dashed line). Although the data points lie clearly closer to the prediction for cold electrons, this alone is not sufficient to decide which regime is realized in the cavity, because of the finite experimental accuracy. An additional criterion is needed.

In order to decide whether the cold or hot electron theory is appropriate for the comparison with the measurements, the electron-electron scattering time $\tau_{e e}$ is compared with the dwell time for electrons inside the cavity. We argue that thermalization is present if $\tau_{D} \gg \tau_{e e}$. The average dwell time is the product of the ballistic flight time across the cavity $\tau_{F} \simeq L / v_{F}$ with the number of scattering events inside the cavity given by the ratio of the cavity size $L$ and the width of the contacts $W=W_{L}+W_{R}=\frac{\lambda_{F}}{2}\left(N_{L}+N_{R}\right)$ :

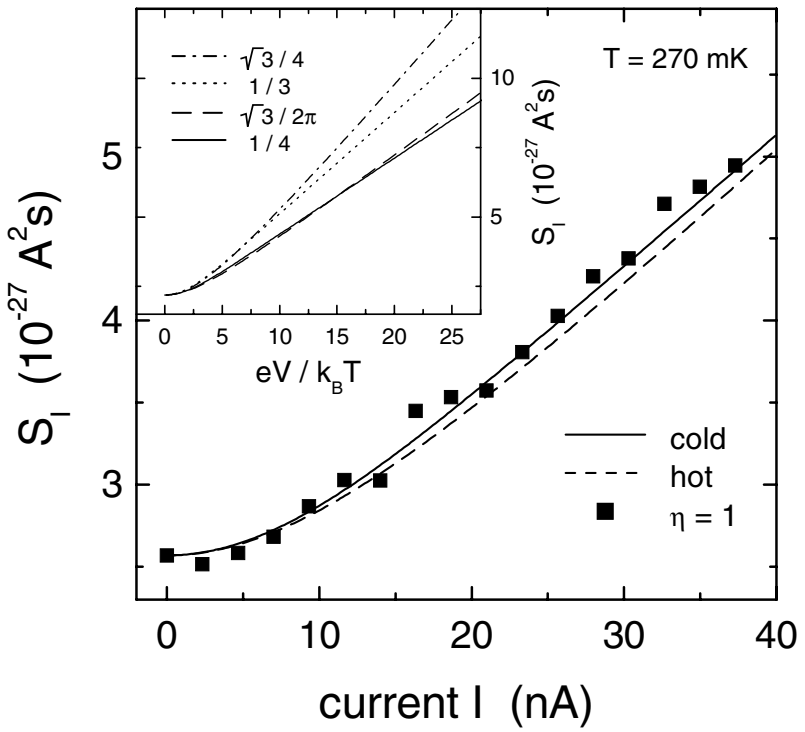

FIG. 3. Shot noise of a symmetric cavity and theoretical predictions for cold (solid line) and hot electrons (dashed line). Inset: comparison of the noise of a chaotic cavity $(1 / 4$ and $\sqrt{3} / 2 \pi)$ with a diffusive wire $(1 / 3$ and $\sqrt{3} / 4)$ for cold and hot electrons.

$$
\tau_{D}=\frac{2 \pi \hbar}{E_{F}}\left(\frac{L}{\lambda_{F}}\right)^{2} \frac{1}{\left(N_{L}+N_{R}\right)} .
$$

The electron-electron scattering rate $\tau_{e e}^{-1}$ in a two dimensional electron system is given by [19]

$$
\tau_{e e}^{-1}=\frac{E_{F}}{2 \pi \hbar}\left(\frac{k_{B} T_{e}}{E_{F}}\right)^{2}\left[\ln \left(\frac{E_{F}}{k_{B} T_{e}}\right)+\ln \left(\frac{2 q}{k_{F}}\right)+1\right]
$$

with the Thomas-Fermi screening wave vector $q=2 m e^{2} / \epsilon_{r} \epsilon_{0} \hbar^{2}$. Because the system is out of equilibrium the temperature $T_{e}$ in Eq. (7) has to be replaced by the effective electron temperature $T_{\text {eff }}$ given by $T_{\text {eff }}=$ $\left(1 / k_{B}\right) \int d \epsilon f_{C}\left(1-f_{C}\right)$ [20]. The ratio $\tau_{D} / \tau_{e e}$ is plotted in the inset of Fig. 4 as a function of $\eta=G_{L} / G_{R}$ for the two different types of cavities taking $\tau_{e e}$ from Eq. (7) for $T_{\text {eff }}$ corresponding to the largest applied voltage $V$ in the experiment. The upper curve belongs to the large cavity $(\simeq 11 \times 8 \mu \mathrm{m})$, where the right contact is nearly closed $\left(G_{R}\right.$ fixed to $\left.G_{0}\right)$. In this case, $\tau_{D} \gg \tau_{e e}$. The lower curve corresponds to the smaller cavity $(\simeq 5 \times 8 \mu \mathrm{m})$ with a 5 times larger opening of the right contact. For this type of cavity we find $\tau_{D}<\tau_{e e}$.

According to this argument we use Eq. (5) valid for hot electrons to fit the noise data obtained for chaotic cavities with $\tau_{D} / \tau_{e e}>1$. The Fano factor $F$ is the only fitting parameter. On the other hand, we use Eq. (3) valid for cold electrons if $\tau_{D} / \tau_{e e}<1$. The Fano factors $F=S / 2 e|I|$ obtained according to this procedure are plotted as a function of the measured $\eta$ for the two different cavities described above. For the solid squares, which belong to the large cavity with nearly closed contacts (large dwell time), we find good agreement with the theoretical Fano 


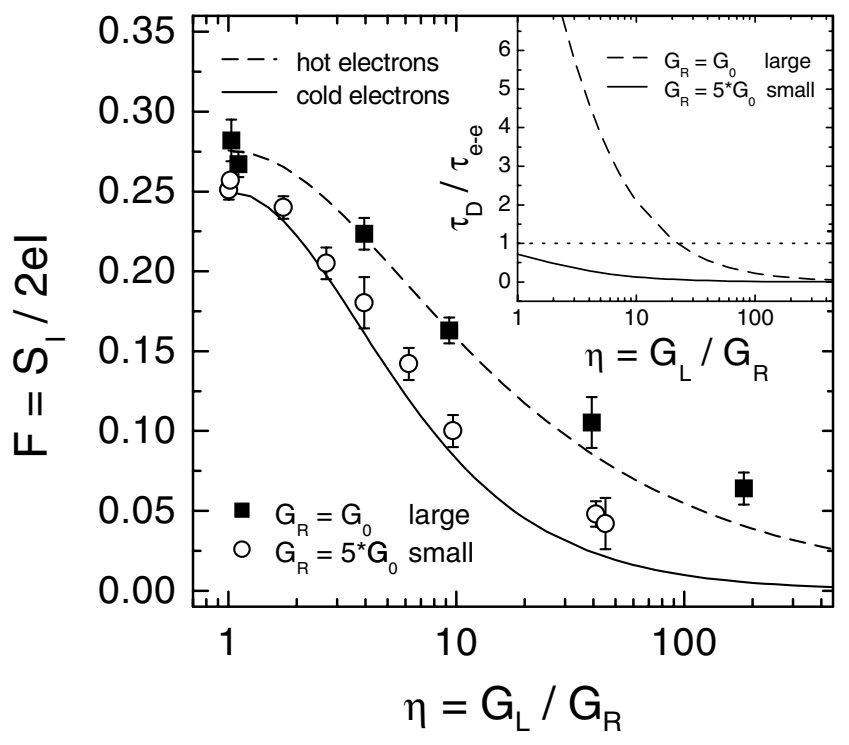

FIG. 4. Fano factor $F \equiv S / 2 e|I|$ vs the symmetry parameter $\eta$ for (open circles) a small cavity with widely opened contacts $\left(\tau_{D}<\tau_{e e}\right)$ and for (solid squares) a large cavity with nearly closed contacts $\left(\tau_{D} \gg \tau_{e e}\right)$. Predictions for cold electrons (solid line) and hot electrons (dashed line). Inset: $\tau_{D} / \tau_{e e}$ vs $\eta$ for the two different types of cavity.

factor for hot electrons given by Eq. (4) (dashed line). The open circles are results for the small cavity with wider opened contacts (small dwell time) which are consistent with the prediction for noninteracting electrons described by Eq. (2). If we use the formula for cold electrons instead of the one for hot electrons to fit the data obtained for the larger cavity, the solid squares move only slightly downwards by $\simeq 0.02-0.03$. They still lie clearly above the open circles, demonstrating that heating is indeed important for the larger cavity. Good agreement between theory and experiment is found for both regimes with the exception of very asymmetric contacts, i.e., $\eta \gg 1$. Here we attribute the deviations to slight mode mixing within the QPCs, which is difficult to avoid [15]. Let us assume, as an example for the data point at $\eta=180$, that two modes instead of one participate in the left contact transmitting, respectively, with $\Gamma_{1}=0.97$ and $\Gamma_{2}=0.03$ instead of $\Gamma_{1}=1.00$ and $\Gamma_{2}=0$. This yields a Fano factor of $\simeq 0.06$ in agreement to what is experimentally observed.

In conclusion, we have experimentally studied shot noise of open chaotic cavities defined by two QPCs in series. In the regime of noninteracting electrons a Fano factor $F=S / 2 e|I|$ of $1 / 4$ has been measured as theoretically predicted for symmetric cavities. The origin of this shot noise is partitioning of the electron wave function by quantum mechanical diffraction inside the cavity. The contacts themselves, which actually define the resistance of the system, do not contribute to noise. In addition, we have also investigated heating effects due to inelastic electron-electron scattering by changing the opening of the contacts as well as the size of the cavity. Similar to other mesoscopic systems heating increases shot noise in agreement with theory. Shot noise in chaotic cavities is a purely quantum phenomenon. It would be interesting to study the crossover from "quantum chaos" to "classical chaos," where shot noise is predicted to be absent [21].

The authors thank Ya. M. Blanter for valuable comments. This work was supported by the Swiss National Science Foundation.

*Present address: Institute for Experimental and Applied Physics, University of Regensburg, D-93040 Regensburg, Germany.

[1] For a recent review, see Ya. M. Blanter and M. Büttiker, Phys. Rep. 336, 1 (2000).

[2] G. B. Lesovik, JETP Lett. 49, 592 (1989).

[3] M. Büttiker, Phys. Rev. Lett. 65, 2901 (1990).

[4] R. A. Jalabert, J.-L. Pichard, and C. W. J. Beenakker, Europhys. Lett. 27, 255 (1994).

[5] Ya. M. Blanter and E. V. Sukhorukov, Phys. Rev. Lett. 84, 1280 (2000).

[6] C. W. J. Beenakker and M. Büttiker, Phys. Rev. B 46, 1889 (1992); K. E. Nagaev, Phys. Lett. A 169, 103 (1992).

[7] R. J. Schoelkopf et al., Phys. Rev. Lett. 78, 3370 (1997).

[8] M. Henny, S. Oberholzer, C. Strunk, and C. Schönenberger, Phys. Rev. B 59, 2871 (1999).

[9] Yu. V. Nazarov, Phys. Rev. Lett. 73, 134 (1994).

[10] E. V. Sukhorukov and D. Loss, Phys. Rev. Lett. 80, 4959 (1998).

[11] B. J. van Wees et al., Phys. Rev. Lett. 60, 848 (1988); D. A. Wharam et al., J. Phys. C 21, L209 (1988).

[12] R. Landauer, IBM J. Res. Dev. 1, 223 (1957).

[13] A. G. Huibers, M. Switkes, C. M. Marcus, K. Campman, and A. C. Gossard, Phys. Rev. Lett. 81, 200 (1998).

[14] Weak localization can be neglected due to residual magnetic flux through the cavity that is larger than $\phi_{0}$.

[15] A. Kumar, L. Saminadayar, D. C. Glattli, Y. Jin, and B. Etienne, Phys. Rev. Lett. 76, 2778 (1996).

[16] L. P. Kouwenhoven et al., Phys. Rev. B 39, 8040 (1989).

[17] K. E. Nagaev, Phys. Rev. B 52, 4740 (1995); V. I. Kozub and A. M. Rudin, Phys. Rev. B 52, 7853 (1995); A.H. Steinbach, J. M. Martinis, and M.H. Devoret, Phys. Rev. Lett. 76, 3806 (1996).

[18] M. J. M. de Jong and C. W. J. Beenakker, in Mesoscopic Electron Transport, edited by L.P. Kouwenhoven, G. Schön, and L.L. Sohn, NATO ASI, Series E, Vol. 345 (Kluwer Academic, Dordrecht, 1996).

[19] G. F. Giuliani and J.J. Quinn, Phys. Rev. B 26, 4421 (1982).

[20] Thermalization has been assumed for $f_{C}$ which could slightly overestimate $\tau_{e e}^{-1}$.

[21] O. Agam, I. Aleiner, and A. Larkin, Phys. Rev. Lett. 85, 3153 (2000). 